\title{
tic\&société
}

Vol. 9, No 1-2 | 1 er semestre 2015 - 2ème semestre 2015

Les religions au temps du numérique

\section{Les travailleurs migrants temporaires au Québec : des migrants connectés?}

Simon Collin, Sirléia Ferreira Da Silva Rosa, Hamid Saffari and Gisèle Charpentier

\section{(2) OpenEdition}

\section{Journals}

Electronic version

URL: http://journals.openedition.org/ticetsociete/1950

DOI: 10.4000/ticetsociete.1950

\section{Publisher}

Association ARTIC

Electronic reference

Simon Collin, Sirléia Ferreira Da Silva Rosa, Hamid Saffari and Gisèle Charpentier, « Les travailleurs migrants temporaires au Québec : des migrants connectés ? », tic\&société [Online], Vol. 9, № 1-2| 1er semestre 2015 - 2ème semestre 2015, Online since 11 January 2016, connection on 02 May 2019. URL : http://journals.openedition.org/ticetsociete/1950; DOI : 10.4000/ticetsociete.1950 


\section{Les travailleurs migrants temporaires au Québec : des migrants connectés?}

Simon Collin, Université du Québec à Montréal

Chaire de recherche du Canada sur les enjeux socioculturels du numérique en éducation

Université du Québec à Montréal (UQAM)

collin.simon@uqam.ca

Sirléia Ferreira Da Silva Rosa

Faculté des sciences de l'éducation

Université de Montréal

sirleia.ferreira.da.silva.rosa@umontreal.ca

\section{Hamid Saffari}

Faculté des sciences de l'éducation

Université du Québec à Montréal (UQAM)

saffari shahrbabaki.hamid@courrier.uqam.ca

\section{Gisèle Charpentier}

Faculté des sciences de l'éducation

Université du Québec à Montréal (UQAM)

charpentier.gisele@courrier.uqam.ca 
Les travailleurs migrants temporaires au Québec : des

migrants connectés?

Simon COLLIN est professeur à la Faculté des sciences de l'éducation de l'Université du Québec à Montréal (UQAM). II est titulaire de la Chaire de recherche du Canada sur les enjeux socioculturels du numérique en éducation et directeur du Centre de recherche interuniversitaire sur la formation et la profession enseignante - Université du Québec (CRIFPE-UQ). Son parcours académique et ses intérêts de recherche portent sur les enjeux socioculturels du numérique en éducation.

Sirléia ROSA est doctorante et chargée de cours à la Faculté des sciences de l'éducation de l'Université de Montréal. Ces expériences académiques et professionnelles lui ont permis de développer une solide expertise en gestion de projets dans le domaine de la formation à distance et des environnements numériques d'apprentissage, et de l'usage des médias sociaux dans les sphères académique et socioculturelle par des migrants et étudiants étrangers.

Hamid SAFFARI est doctorant à la Faculté des sciences de l'éducation à l'Université du Québec à Montréal (UQAM). II est également coordinateur de la Chaire de recherche sur les enjeux socioculturels du numérique en éducation. Son expertise porte sur l'utilisation des technologies en éducation, notamment l'apprentissage mobile. II a plusieurs communications et publications scientifiques à son actif, tant sur le plan national qu'international.

Gisèle CHARPENTIER est candidate à la maitrise à la Faculté des sciences de l'éducation de l'Université du Québec à Montréal (UQAM) et membre du CRIFPE. Ses expériences professionnelles l'ont amenée à développer une expertise en enseignement du français, langue seconde et langue étrangère, dans le milieu collégial et universitaire. Ses intérêts de recherche portent sur les besoins langagiers et d'intégration linguistique des étudiants et des migrants étrangers, notamment des travailleurs migrants temporaires.

\section{Résumé}

Les études sur les usages des technologies de l'information et de la communication (TIC) par les migrants ont fait l'objet d'un intérêt scientifique croissant ces dernières années. En revanche, peu d'études se sont penchées sur le cas particulier des travailleurs migrants temporaires, qui vivent annuellement des phases de non-migration et des phases de migration. Aussi, 


\section{Simon COLLIN, Sirléia ROSA, Hamid SAFFARI et Gisèle CHARPENTIER}

l'objectif de cette étude est de mieux comprendre l'accès et les usages des TIC par cette catégorie de migrants au Québec. Les résultats obtenus au moyen d'une enquête auprès de 24 travailleurs agricoles mexicains et guatémaltèques indiquent que le fait de migrer correspond à une réduction de la diversité des usages des TIC pour une majorité des participants. Toutefois, une minorité d'entre eux semblent utiliser au Québec une ou des TIC (le téléphone cellulaire principalement) qu'ils n'utilisaient pas dans leur société d'origine. En outre, l'éducation et la nationalité ressortent comme deux variables associées aux variations d'usage des TIC au sein de cet échantillon. Des pistes d'explication de ces résultats sont explorées en référence à la littérature scientifique du domaine des TIC et de la migration.

Mots-clés: usages des technologies de l'information et de la communication; travailleurs migrants temporaires; empowerment; inégalités socionumériques.

\section{Summary}

Information and communications technology (ICT) use by migrants is a growing research field. However, so far, few studies have focused on temporary miarants workers, who experience periods of migration and periods of non-migration on a vearly basis. The objective of this paper is to gain a better understandina of ICT use bv 24 Mexican and Guatemalan aqricultural temporary migrant workers in Quebec. The results show that migration tends to reduce the diversity of ICT use for most of the participants. However, a few of them seem to use one or more ICT (mainly the cell phone) in Quebec that they did not use in the source society. Moreover, education and citizenship are two variables that are linked to ICT use in this sample. These results are discussed in regards to the scientific literature on ICT and migration.

Key words: information and communication technology use; temporary migrant workers; empowerment; sociodigital inequalities.

\section{Resumen}

Los estudios sobre el uso de las tecnologías de información y comunicación (TIC) para migrantes han sido objeto de un creciente interés científico en los últimos años. En cambio, pocos 
Les travailleurs migrants temporaires au Québec : des

migrants connectés?

estudios se han realizado en el caso específico de los trabajadores migrantes temporales, que viven cada año periodos intermitentes de migración a nuevos entornos físicos y culturales. El presente estudio tiene como propósito mejorar la comprensión del acceso y uso de las TIC por parte de esta categoría de migrantes en Quebec. Los resultados obtenidos por medio de un cuestionario a 24 trabajadores agrícolas mexicanos y guatemaltecos indican que el hecho de migrar equivale a una reducción en la diversidad de usos de las TIC para la mayoría de los participantes. Sin embargo, una minoría de ellos parece utilizar una TIC principalmente, el teléfono celular, que no utilizan en su lugar de origen. Entre otros resultados obtenidos, dos variables resaltan en el uso de las TIC, el nivel de educación y nacionalidad de los encuestados. En el presente estudio, exploramos varias hipótesis para explicar estos resultados con referencia a la literatura científica publicada sobre las TIC y la migración.

Palabras claves: usos de las tecnologías de información y comunicación; trabajadores migrantes temporales; empowerment; desigualdad digitales. 


\section{Simon COLLIN, Sirléia ROSA, Hamid SAFFARI et Gisèle CHARPENTIER}

\section{Introduction}

L'étude des usages des technologies de l'information et de la communication (TIC) par les migrants est relativement récente et attire un nombre croissant de recherches théoriques et empiriques (Collin, 2012; Mattelart, 2009), notamment depuis le texte fondateur de Diminescu (2005) sur le « migrant connecté ». Cette nouvelle figure du migrant repose sur le constat que les TIC sont de plus en plus intégrées au processus migratoire, de sorte que ce dernier, qui était auparavant perçu comme une série de ruptures, serait à envisager davantage en termes de continuité. En effet, dès la phase prémigratoire, les TIC seraient susceptibles de faciliter, à distance, la préparation de la migration et d'accélérer le processus d'intégration des migrants dans la société d'accueil. En phase post-migratoire, elles permettraient aux migrants de maintenir et de générer de nouveaux liens avec la société d'origine, de s'engager dans des activités économiques, sociales et politiques transnationales et de forger des identités cosmopolites (Bayon, 2009; Diminescu, 2002; Nedelcu, 2002, 2009; Oosterbaan, 2010; Scopsi, 2004, 2009; Mattelart, 2009). Parallèlement, les études sur les inégalités socionumériques (par exemple, DiMaggio et al., 2004) montrent que, au-delà du potentiel attribué aux TIC, en tirer profit dépend notamment du profil socioculturel et migratoire des individus. Dans ce contexte, l'objectif de cette étude exploratoire est de mieux comprendre l'accès et les usages des TIC par les travailleurs migrants temporaires au Québec, notamment au regard des thèses d'empowerment et d'inégalités socionumériques.

Pour poser le contexte de l'étude, nous commençons par décrire le profil et les conditions de travail des travailleurs migrants temporaires au Canada, en ciblant ceux du secteur agricole qui font l'objet de cette recherche. Nous dressons ensuite un bref portrait du domaine des TIC et de la migration, avant de l'envisager selon deux thèses pertinentes et complémentaires pour notre objet d'étude, soit la thèse de l'empowerment et celle des inégalités socionumériques, sans pour autant l'y réduire. S'ensuivent la présentation de la méthodologie et des résultats, puis la discussion de ces derniers à partir de la littérature du domaine des TIC et de la migration. 
Les travailleurs migrants temporaires au Québec : des

migrants connectés?

\section{Les travailleurs migrants temporaires au Canada : le cas du secteur agricole}

II existe actuellement différents programmes ayant pour but d'aider les exploitants agricoles canadiens, qui manquent de main-d'œuvre à certaines périodes de l'année, à recruter temporairement des travailleurs étrangers. Le premier d'entre eux s'intitule le Programme des Travailleurs Agricoles Saisonniers (PTAS) et consiste en un accord intergouvernemental entre le Canada et les Caraïbes mis en place à partir de 1966, puis entre le Canada et le Mexique à partir de 1974 (Ressources humaines et développement des compétences Canada, 2009). En 2008, ce programme a concerné plus de 27700 travailleurs, dont 3300 au Québec. À partir de 2002 s'est ajouté le Programme pour Travailleurs Étrangers Temporaires (PTET), non spécifique au secteur agricole, qui permet aux entreprises canadiennes de « recruter les travailleurs migrants temporaires dans le pays de leur choix sans que des mécanismes de contrôle limitent leur processus de sélection, comme c'est le cas dans le cadre du PTAS » (Amar, Roberge, LaRue, Gélineau et Leanza, 2009, p. 4). Les exploitants agricoles canadiens peuvent donc embaucher des travailleurs agricoles temporaires dans l'un ou l'autre de ces programmes (Bellerose et Yorn, 2013).

Ces travailleurs sont principalement des pères de famille issus de régions rurales pauvres. Ils sont logés (gratuitement ou non, dépendamment du programme) par l'exploitation canadienne où ils travaillent (Reyes Trigos, 2006). Leur motivation à participer à ces programmes est avant tout financière, puisque leur salaire au Canada est de cinq à six fois plus élevé que dans leur région d'origine (Preibisch, 2007), ce qui permet en retour d'améliorer les conditions de vie de leur famille grâce aux envois de fonds (Binford, 2006).

Malgré les avantages qu'ils tirent de ces programmes, les travailleurs rencontrent plusieurs problèmes liés à leurs conditions de vie. En premier lieu, leur emploi et leur lieu de travail sont prédéterminés et difficiles à changer (Basok, 2000; Elgersma, 2007) dans la mesure où ils ne sont pas autorisés à rester sur le sol canadien en dehors des modalités du programme. Des chercheurs du domaine les décrivent également à risque d'exclusion sociale en raison de leur statut temporaire, de leur manque d'accès à l'information sur les protections existantes, de leur faible mobilité durant leur séjour, de l'obstacle de la langue, et parfois du mépris des habitants 


\section{Simon COLLIN, Sirléia ROSA, Hamid SAFFARI et Gisèle CHARPENTIER}

(Elgersma, 2007; Preibisch, 2007). Finalement, la peur de perdre leur emploi, et, du même coup, les surplus financiers qu'ils génèrent par rapport à leur région d'origine, poussent les travailleurs migrants temporaires à être très conciliants avec leurs employeurs.

La condition des travailleurs migrants temporaires est donc appréciée de façon partagée dans les recherches empiriques qui s'y penchent. Nous proposons de contribuer à la discussion en approchant cet objet d'étude sous l'angle des usages des TIC. La section suivante permet de préciser la pertinence d'étudier les usages des TIC par les travailleurs migrants temporaires.

\section{Les éléments conceptuels}

Nous commençons par donner un bref aperçu du domaine des TIC et de la migration, avant de nous focaliser sur deux thèses susceptibles d'éclairer notre objet d'étude, sans qu'il y soit réduit pour autant, soit la thèse de l'empowerment et celle des inégalités socionumériques.

\subsection{Un aperçu du domaine des TIC et de la migration}

Les recherches sur les usages des TIC par les migrants et les minorités ethniques se sont structurées à partir de la deuxième moitié des années 1990 (Mattelart, 2009) bien que ce soit réellement « à partir de l'année 2000 que les minorités ethniques profitent de l'élan permis par l'arrivée massive des technologies sur Internet pour investir le web » (Rigoni, 2010). Les principales disciplines impliquées dans l'étude des usages des TIC par les migrants et les minorités ethniques sont la sociologie, la communication et l'anthropologie, ainsi que certaines disciplines connexes comme la géographie, la démographie et les sciences informatiques. Étant donné la diversité d'approches possibles, "the study on ICT and migrants, today, is a genuinely interdisciplinary field of research » (Borkert et al., 2009, p. 18). 
Les travailleurs migrants temporaires au Québec : des

migrants connectés?

\subsection{Les usages des TIC par les migrants et les minorités ethniques}

Plusieurs concepts peuvent être exploités pour éclairer les usages des TIC par les migrants. Parmi eux, le concept de transnationalisme, défini comme "sustained, cross-boarder, relationships, patterns of exchange, affiliations and social formation spanning nation-states » (Vertovec, 2009, p. 2) a été largement utilisé pour situer le rôle des TIC au sein des réseaux migratoires et entre les diasporas et leurs sociétés d'origine, tant sur le plan des relations interpersonnelles (notamment familiales et communautaires, Hannaford, 2014), qu'économiques (Portes, Haller et Guarnizo, 2002) et politiques (Nedelcu, 2009). Bien que le concept de transnationalisme soit relativement bien défini et circonscrit, certains auteurs (Fibbi et d'Amato, 2008; Garduno, 2003) notent que son opérationnalisation méthodologique et ses manifestations empiriques restent difficiles à établir. D'autres soulignent que la popularité de ce concept tend à exagérer la dimension transnationale dans la vie des migrants, et à occulter, par contre coup, les processus mis à œuvre par les migrants relativement à leur intégration, comme le relève Mattelart pour les cas des usages d'Internet:

«Tendant à se concentrer sur les connexions qu'internet contribue à établir entre les migrants et leur patrie d'origine, les recherches sur les TIC et les migrations peinent à analyser la manière dont les migrants articulent fréquentation des sites relatifs au pays quitté avec ceux relatifs au pays de résidence (pour n'évoquer que ceuxlà !) » (2009, p. 32).

D'autres études du domaine des TIC et de la migration, sans réfuter la dimension transnationale des usages des TIC par les migrants, adoptent une posture davantage critique. Elles tentent notamment d'apprécier les bénéfices qu'en tirent les migrants et les conditions nécessaires à l'apparition de ces derniers. Nous illustrons cette perspective en nous focalisant sur deux thèses, qui émergent des recherches du domaine et des discours des chercheurs. La première pose les usages des TIC par les migrants et les minorités ethniques comme un moyen d'empowerment de ces populations. C'est généralement le cas d'études ou d'auteurs qui relèvent le rapport étroit que les migrants et les minorités entretiennent avec les TIC, et le potentiel présumé ou les avantages empiriquement observés qu'ils en retirent. Ce rapport étroit avec les TIC s'expliquerait par 


\section{Simon COLLIN, Sirléia ROSA, Hamid SAFFARI et Gisèle CHARPENTIER}

différents facteurs: l'âge moyen des migrants (plus jeune que celui de la population locale), qui serait associé à une plus grande familiarité avec les TIC; la nécessité de rester régulièrement en contact avec la famille et les amis; et le besoin de se connecter à Internet pour des raisons d'éducation et d'employabilité dans la société d'accueil (Codagnone et Kluzer, 2011; Kluzer, Hache et Codagnone, 2008). La seconde thèse situe les usages des TIC comme une manifestation d'inégalités socionumériques. II s'agit généralement d'études ou d'auteurs qui soulignent les disparités dans les usages des TIC entre les migrants et les minorités ethniques et la société d'accueil. Ces disparités étant articulées autour de dimensions sociodémographiques (par exemple le sexe et l'âge), socioéconomiques (le revenu et le niveau d'éducation) et socioculturelles (le statut migratoire et l'ethnicité), elles sont généralement interprétées comme le reflet d'inégalités sociales plus larges, dans l'idée que les TIC reproduisent, plutôt qu'elles ne réduisent, les inégalités sociales préexistantes (DiMaggio et al., 2004).

Bien qu'elles puissent paraitre contradictoires en apparence, ces deux thèses ne sont pas mutuellement exclusives. En effet, on imagine facilement que les TIC puissent à la fois faciliter certains aspects de la condition des migrants et des minorités ethniques (par exemple, en offrant des possibilité de contacts plus fréquents, plus riches et plus diversifiés avec la société d'origine) tout en conservant dans leurs grandes lignes les inégalités d'accès et d'usages des TIC présentes dans la société d'accueil. Á ce stade-ci de l'avancement des connaissances, il nous apparait donc que la relation entre ces deux thèses reste à explorer au regard des profils socioculturels hétérogènes des migrants et des minorités ethniques (Caidi et Allard, 2005). II s'agit précisément de l'objectif de cet article qui cible un type particulier de migrants, en l'occurrence les travailleurs migrants temporaires du Québec dont les usages des TIC ont peu été étudiés à notre connaissance. Les TIC peuvent permettre à ces derniers de rester en contact avec leurs proches, en se substituant aux interactions en face à face, que la migration rend impossibles. En effet, les réseaux sociaux, le courriel, les téléphones cellulaires (notamment au moyen des cartes d'appel international) et la téléphonie IP (via Skype par exemple) deviennent autant d'opportunités permettant de compenser la diminution des contacts en présentiel qui existaient dans la phase prémigratoire (Ros, 2010). D'après Hiller et Franz (2004), les TIC sont particulièrement utilisées par les migrants au début 
Les travailleurs migrants temporaires au Québec : des

migrants connectés?

du parcours migratoire puisque leurs relations avec la société d'origine sont «encore récentes et dynamiques» (p. 743). II serait donc attendu que les travailleurs migrants temporaires mettent à profit les diverses opportunités relationnelles permises par les TIC dans la mesure où leur parcours migratoire se caractérise par de courtes durées, généralement répétées sur plusieurs années. De plus, les fonds gagnés par l'intermédiaire du PTAS leur fournit a priori l'occasion d'investir dans l'équipement technologique visant à pallier leur absence durant les phases migratoires. Pourtant, les conditions de vie précaires des travailleurs migrants temporaires (voir section 1) invitent à questionner la possibilité pour ces migrants en particulier de capitaliser sur les TIC pour les usages mentionnés ci-dessus, de sorte que la présence de manifestations d'inégalités socionumériques n'est pas à exclure.

\section{Les éléments de méthodologie}

\subsection{Les participants}

L'étude s'est déroulée dans une ferme québécoise spécialisée dans la production de divers légumes et accueillant depuis plusieurs années des travailleurs migrants temporaires du Mexique et du Guatémaltèque. Au moment de la collecte de données (mai 2012), 24 travailleurs migrants temporaires hispanophones (14 Mexicains et 10 Guatémaltèques) représentaient l'essentiel des ouvriers agricoles. (Leur profil sociodémographique est détaillé dans la section 4.1. des résultats.)

\subsection{La collecte de données}

La collecte des données a consisté en un questionnaire et trois entrevues de groupe auprès des 24 travailleurs migrants temporaires. Les résultats présentés ici sont issus du questionnaire uniquement. Les questions étaient fermées et ont été sélectionnées et adaptées à partir d'études ou de questionnaires existants, tels que l'étude de Livingstone et Helsper (2007) et le Canadian Internet Use Survey (Statistic Canada, 2011), suivant leur pertinence au regard de l'objectif poursuivi. D'autres aspects ont été ajoutés, tel que l'axe comparatif des usages des TIC entre la société d'origine et la société d'accueil. Le questionnaire a ensuite été validé par le 


\section{Simon COLLIN, Sirléia ROSA, Hamid SAFFARI et Gisèle CHARPENTIER}

personnel administratif de la ferme, après acceptation des termes éthiques du projet (notamment en ce qui concerne le respect de l'anonymat et de la confidentialité des propos recueillis auprès de leurs travailleurs), puis traduit en espagnol et mis en page de façon à ce qu'il soutienne au mieux la compréhension des participants. Finalement, la passation des questionnaires a été effectuée sur papier avec le soutien oral d'une locutrice parlant espagnol, de façon à ce que les questions soient lues oralement et que les participants puissent poser des questions si nécessaire.

\subsection{Les analyses de données}

Nous avons commencé par procéder à des analyses descriptives afin de dresser un portrait des principaux usages des TIC par les participants. En vue des analyses statistiques inférentielles, et étant donné la faible taille de l'échantillon, il a été décidé de regrouper les données en ne conservant pour chaque question que deux valeurs possibles, de façon à obtenir une distribution relativement équilibrée des réponses. Par exemple, nous avons regroupé les données relatives à l'âge suivant les deux valeurs suivantes: 18-32 ans (avec 11 participants); 33-48 ans (avec 13 participants).

Nous avons ensuite cherché à expliquer quelles variables indépendantes (âge, niveau d'éducation, nationalité, nombre de membres familiaux à charge et zone d'habitation) influencent la diversité des usages des TIC par les participants dans leur société d'origine, et leur diversité des usages des TIC au Québec. La diversité des usages des TIC est établie à partir du nombre de TIC utilisées par chaque participant, indépendamment des TIC en tant que telles (voir Figures 3 et 4). II s'agit d'une variable fiable à partir de laquelle étudier les usages des TIC, comme en attestent plusieurs études (voir, par exemple, Hargittai, 2010; Helsper et Eynon, 2010 ; Livingstone et Helsper, 2007; Wei, 2012). Afin d'approfondir les résultats de cette étude exploratoire, nous avons également procédé à des corrélations, tout en retenant que les résultats sont à prendre avec prudence étant donné la petite taille de l'échantillon. 
Les travailleurs migrants temporaires au Québec : des

migrants connectés?

\section{Les résultats}

Dans cette section, nous commençons par donner un aperçu du profil sociodémographique et migratoire des participants à l'étude. Nous dressons ensuite un portrait général des usages des TIC par les participants, avant de nous pencher plus particulièrement sur la diversité des usages des TIC et les variables socioculturelles qui l'influencent.

\subsection{Un aperçu du profil des participants}

Les participants à l'étude étaient des hommes, âgés de 22 à 46 ans. Quatorze sont mexicains alors que les 10 autres sont Guatémaltèques. Dans les deux cas, ils viennent majoritairement de zones rurales $(66,7 \%)$ ou de zones semi-urbaines (25\%). Ils sont en grande majorité mariés $(71 \%)$ ou en union libre $(17 \%)$ et seulement un d'entre eux n'avait pas d'enfant à charge au moment de la collecte de données. Le niveau d'éducation est relativement divisé. En effet, 10 participants ont bénéficié d'une scolarité primaire, alors que 13 autres ont reçu une éducation secondaire. Un seul participant a poursuivi sa scolarité au niveau postsecondaire.

Un dernier élément à mentionner concerne le nombre d'années d'expérience en tant que travailleurs migrants temporaires. Seulement trois participants le sont pour la première fois. À l'inverse, une majorité de répondants a une expérience de deux à quatre ans (15) en tant que travailleurs migrants temporaires, et de cinq à huit ans (4) ou de neuf ans et plus (2).

De cet aperçu du profil sociodémographique et migratoire des participants, retenons que ces derniers semblent représentatifs de la population des travailleurs migrants temporaires, telle que décrite précédemment. En effet, à l'instar de cette population, les participants à l'étude sont principalement des pères de famille en provenance de régions rurales pauvres, qui ont un niveau d'éducation relativement faible, et qui ont plusieurs années d'expérience en tant que travailleurs migrants temporaires. 


\section{Simon COLLIN, Sirléia ROSA, Hamid SAFFARI et Gisèle}

CHARPENTIER

\subsection{Un portrait général des usages des TIC}

Commençons par présenter les différentes TIC auxquels les participants ont régulièrement recourt. La figure 1 indique les TIC utilisées au moins une fois par mois dans la société d'origine, alors que la figure 2 présente celles utilisées lorsque les participants sont au Québec. Ces deux figures montrent des tendances similaires. Ainsi, le téléphone cellulaire sans accès à Internet est de loin la technologie la plus utilisée par les participants, tant dans leur société d'origine (16) qu'au Québec (17), à laquelle s'ajoute de façon moindre le téléphone cellulaire intelligent (5 dans la société d’origine; 4 au Québec).

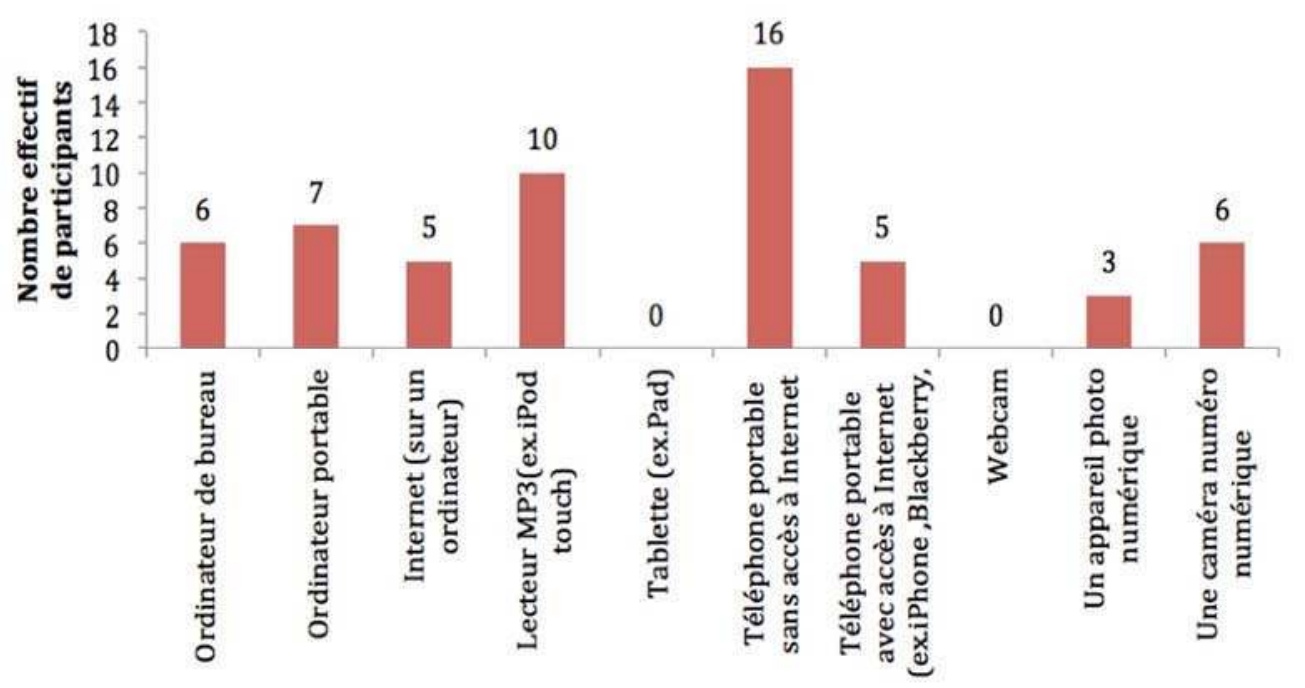

Figure 1. Usage des TIC par les participants dans leur société d'origine (au moins une fois par mois). 
Les travailleurs migrants temporaires au Québec : des migrants connectés?



Figure 2. Usage des TIC par les participants au Québec (au moins une fois par mois).

Par ailleurs, les participants répondent privilégier le téléphone cellulaire (20) et le téléphone fixe (7), au moyen d'une carte prépayée d'appel international, pour contacter leurs proches dans leur société d'origine.

Les autres TIC utilisées dans la société d'origine le sont également au Québec, mais dans des proportions moins importantes. Ainsi, le lecteur MP3 et l'ordinateur portable sont respectivement les deuxième et troisième TIC les plus fréquemment utilisées par les participants, bien que ce soit davantage le cas dans la société d'origine (respectivement, 10 et 7) qu'au Québec (respectivement, 6 et 4). De plus, certaines TIC utilisées dans la société d'origine, telles que l'ordinateur de bureau (6) et Internet (5), ne le sont quasiment pas lorsque les participants sont au Québec (respectivement, 1 et 2).

Ces résultats nous invitent à penser que le fait de migrer ne semble pas entrainer un accroissement des usages des TIC. Au contraire, de manière globale, on constate que les participants sont plus nombreux à utiliser les TIC lorsqu'ils sont dans leur société d'origine que lorsqu'ils sont au Québec. 


\section{Simon COLLIN, Sirléia ROSA, Hamid SAFFARI et Gisèle CHARPENTIER}

\subsection{La diversité et l'hétérogénéité des usages des TIC}

Les résultats précédents peuvent être repris sous l'angle de la diversité des usages des TIC, qui consiste à mesurer, pour chaque participant, combien de TIC il utilise. Deux résultats principaux sont à mentionner. En premier lieu, il apparaît que les participants ont des usages des TIC plus diversifiés dans leur société d'origine par comparaison avec les usages qu'ils développent au Québec (figures 3 et 4). En effet, lorsqu'ils sont dans leur société d'origine, six participants utilisent au moins une technologie par mois alors que quatorze d'entre eux en utilisent de deux à sept. En revanche, au Québec, la majorité des participants (15) n'utilisent qu'une technologie par mois, laquelle correspond sans doute au téléphone cellulaire, si l'on se reporte à la figure 2, alors que sept d'entre eux seulement utilisent de deux à quatre TIC. En second lieu, les participants sont au nombre de quatre à n'utiliser aucune technologie dans leur société d'origine, alors que ce chiffre décroit à un lorsqu'ils sont au Québec.

D'une manière globale, les participants semblent donc avoir des usages des TIC plus diversifiés (en termes de nombre d'usages des TIC) et hétérogènes (en termes de différences entre participants) lorsqu'ils sont dans leur société d'origine, alors que ces usages deviennent plus restreints et davantage homogènes lorsqu'ils sont au Québec. 
Les travailleurs migrants temporaires au Québec : des migrants connectés?

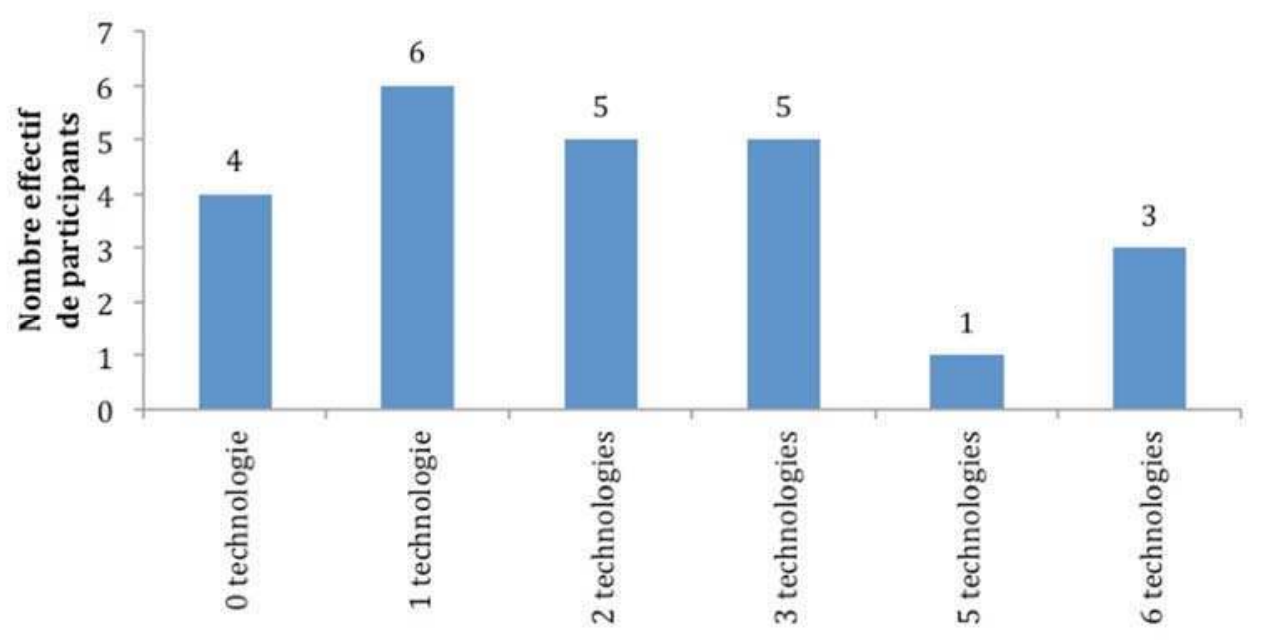

Figure 3. Diversité des usages des TIC par les participants dans leur société d'origine (par mois).

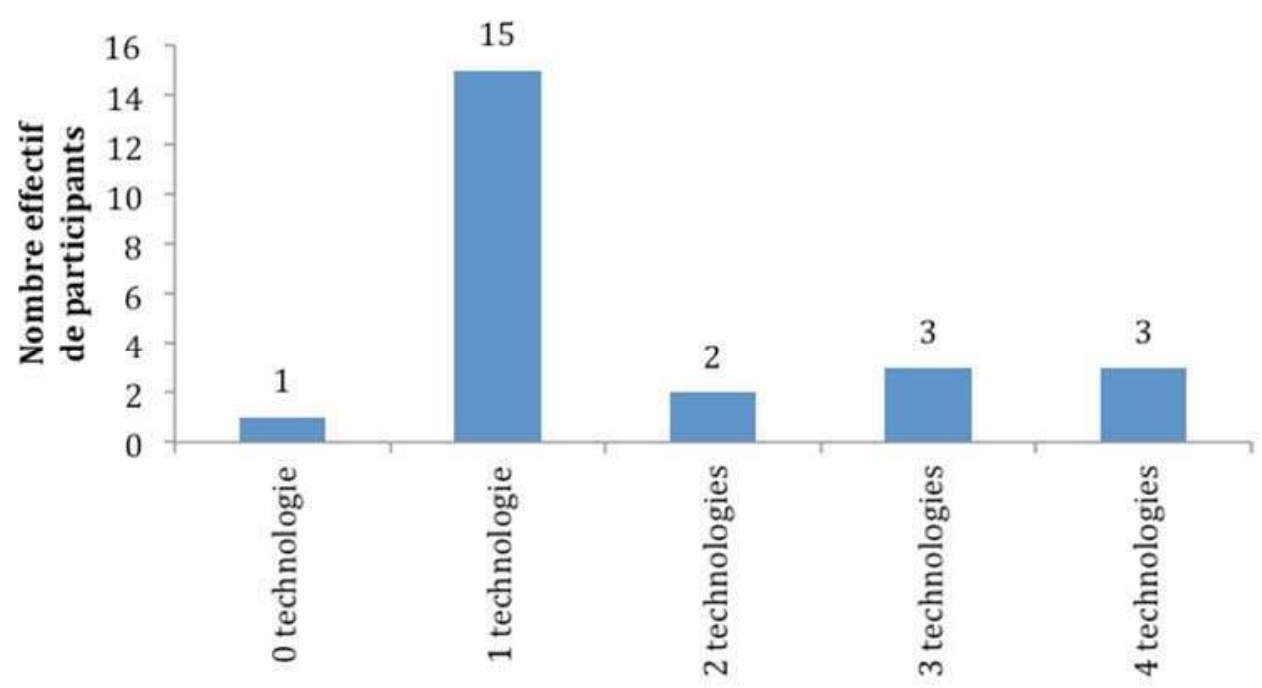




\section{Simon COLLIN, Sirléia ROSA, Hamid SAFFARI et Gisèle CHARPENTIER}

Figure 4. Diversité des usages des TIC par les participants au Québec (par mois).

Deux tendances émergent des résultats ci-dessus. D'un côté, le fait de migrer réduit la diversité des usages des TIC d'une majorité de participants, ce qui rejoint les résultats et interprétations des figures 1 et 2, qui montrent que les TIC utilisées dans la société d'origine le sont également au Québec, mais dans des proportions moins importantes. Parallèlement, la migration amène une minorité de participants à utiliser au Québec une ou des TIC qu'ils n'utilisaient pas dans leur société d'origine, notamment le téléphone cellulaire. Ces deux mouvements, l'un décroissant (en nombre d'usages des TIC) et majoritaire (en nombre de participants), l'autre croissant et minoritaire, ont pour effet d'homogénéiser davantage les usages des TIC lors de la migration que dans la société d'origine.

Dans la perspective exploratoire de l'étude, nous avons recouru à des corrélations, tout en rappelant que les résultats sont à prendre avec prudence étant donné la petite taille de l'échantillon. Nous avons pris comme variables la diversité des usages des TIC dans la société d'origine et la diversité des usages des TIC au Québec. Les résultats indiquent que ces deux variables sont effectivement associées de manière significative (voir annexe) et que leur force d'association est jugée élevée. Autrement dit, plus les travailleurs migrants temporaires ont des usages des TIC diversifiés dans leur société d'origine, plus ils ont des usages diversifiés, bien que plus restreints, au Québec.

En rapport aux résultats évoqués plus haut, bien que le fait de migrer puisse entrainer une certaine homogénéisation des usages des TIC parmi les participants, il ne semble pas suffisant pour "gommer » l'hétérogénéité des usages des TIC préexistants dans la société d'origine. Ainsi, l'hétérogénéité, qui caractérise les usages des TIC par les participants avant la migration, semble persister durant le processus migratoire, bien qu'elle soit moins grande. Se pose alors la question de savoir à quoi est due cette hétérogénéité.

\subsection{Les variables socioculturelles à l'œuvre dans I'hétérogénéité des usages des TIC}

Nous avons cherché à expliquer quelles variables indépendantes (âge, niveau d'éducation, nationalité, nombre de 
Les travailleurs migrants temporaires au Québec : des

\author{
migrants connectés?
}

membres familiaux à charge et zone d'habitation) influencent la diversité des usages des TIC par les participants dans leur société d'origine, et leur diversité des usages des TIC au Québec. Les résultats ci-dessous portent uniquement sur la nationalité et le niveau d'éducation, car il s'agit des deux seules variables qui ont fait état de différences significatives au sein de notre échantillon.

La nationalité constitue la première variable faisant état d'une différence significative entre la diversité des usages des TIC par les participants dans leur société d'origine (voir annexe). Les participants mexicains ont des usages des TIC plus diversifiés que les participants guatémaltèques.

La deuxième variable faisant état d'une différence significative au sein de notre échantillon concerne le niveau d'éducation (voir annexe). On observe en effet que les participants ayant bénéficié d'une scolarité secondaire ou postsecondaire ont des usages des TIC plus diversifiés dans leur société d'origine que les participants ayant reçu une scolarité primaire.

Comme résultats additionnels, il est intéressant de noter que ces deux variables (nationalité et niveau d'éducation) restent importantes lorsqu'on considère la diversité des usages des TIC au Québec, bien que l'éducation n'apparaisse plus significative. Nous pouvons donc avancer que la nationalité et le niveau d'éducation sont deux variables particulièrement déterminantes de la diversité des usages des TIC dans notre échantillon, que ces usages aient lieu dans la société d'origine des participants ou au Québec, alors que l'âge, le nombre de membres familiaux à charge et la zone d'habitation n'interviennent pas de manière significative.

\title{
5. Discussion et conclusion
}

Pour rappel, l'objectif de cette étude exploratoire est de mieux comprendre l'accès et les usages des TIC par les travailleurs migrants temporaires, notamment au regard des thèses de l'empowerment et/ou des inégalités socionumériques.

Concernant les résultats principaux obtenus, commençons par rappeler que le profil sociodémographique et migratoire des participants à l'étude semble être vraisemblablement en phase avec celui de la population des travailleurs migrants temporaires du secteur agricole (c.-à-d., des pères de famille en provenance de régions rurales pauvres, qui ont un niveau d'éducation 


\section{Simon COLLIN, Sirléia ROSA, Hamid SAFFARI et Gisèle CHARPENTIER}

relativement faible, et qui ont plusieurs années d'expérience en tant que travailleurs migrants temporaires). Pourtant, le faible échantillon de participants interdit toute généralisation des résultats statistiques obtenus.

Les résultats statistiques descriptifs (Figures 1 et 2) semblent indiquer des usages des TIC relativement limités. Le téléphone cellulaire (principalement sans accès à Internet) est de loin la technologie la plus partagée parmi nos participants, alors que l'ordinateur (portable ou fixe) est peu utilisé. Ces résultats sont cohérents avec les tendances observées mondialement. Ainsi, I'International Telecommunication Union (ITU) rapporte que les populations des pays en voie de développement, dans lesquels figurent le Mexique et le Guatémaltèque d'après le classement proposé par le Fonds Monétaire international (2013), sont équipées en téléphone cellulaire à hauteur de $89 \%$, alors que seulement $31 \%$ d'entre elles utilisent Internet, contre $77 \%$ pour les populations des pays développés (ITU, 2013). En outre, nos résultats indiquent que les participants sont plus nombreux à utiliser les TIC lorsqu'ils sont dans leur société d'origine que lorsqu'ils sont au Québec. Le fait de migrer ne semble donc pas générer un accroissement des usages des TIC chez les participants, de sorte qu'un empowerment au moyen des TIC semble peu probable, contrairement aux études démontrant un rôle important des TIC pour les migrants et les minorités ethniques (voir, par exemple, Bayon, 2009; Oosterbaan, 2010; Scopsi, 2009). Ce résultat semble toutefois cohérent avec l'étude de Komito et Bates (2011), qui ne rapporte aucun accroissement des usages d'Internet après la migration.

Nous avons repris ces résultats sous l'angle de la diversité des usages des TIC. À cet égard, le fait de migrer semble correspondre à une réduction de la diversité des usages des TIC pour une majorité des participants à l'étude. Toutefois, une minorité de participants semblent utiliser au Québec une ou des TIC (le téléphone cellulaire principalement) qu'ils n'utilisaient pas dans leur société d'origine.

Ces deux mouvements, l'un décroissant (en nombre d'usages des TIC) et majoritaire (en nombre de participants), l'autre croissant et minoritaire, ont pour effet une homogénéisation apparente des usages des TIC par les participants lorsqu'ils sont en situation de migration. Cependant, nos analyses montrent que plus les participants ont des usages des TIC diversifiés dans leur société d'origine, plus ils ont des usages diversifiés, bien que plus restreints, au Québec. Autrement dit, l'hétérogénéité qui 
Les travailleurs migrants temporaires au Québec : des

migrants connectés?

caractérise les usages des TIC par les participants avant la migration semble persister durant la migration, de sorte que la tendance à l'homogénéisation avancée plus haut n'est pas vérifiable à partir des résultats exploratoires dont nous disposons.

De façon complémentaire, la nationalité et le niveau d'éducation ressortent comme les deux variables socioculturelles déterminantes de la diversité des usages des TIC au sein de notre échantillon dans la société d'origine et au Québec.

L'importance de la nationalité peut a priori s'expliquer par la différence de niveau de développement infrastructurel entre le Guatemala et le Mexique. En effet, différents indices, tels que l'indice de développement humain (PNUD), montrent des écarts de développement importants entre le Mexique et le Guatemala ${ }^{1}$. En outre, plusieurs études ont souligné le lien entre le développement infrastructurel d'une population et son degré d'adoption des TIC (Chinn et Fairlie, 2007; Hanafizadeh, Saghaei et Hanafizadeh, 2009). Nous pouvons donc avancer l'idée que la nationalité serait une variable reflétant indirectement le niveau de développement infrastructurel.

Le niveau d'éducation a régulièrement été mentionné comme un facteur expliquant les variations interindividuelles (Helsper et Eynon, 2010; Livingstone et Helsper, 2007; DiMaggio et al., 2004) et internationales (Mrad, 2006; Vicente et Lopez, 2006) des usages des TIC. Plusieurs raisons permettent d'expliquer l'importance de l'éducation dans l'accès et les usages des TIC. En premier lieu, elle constitue un des principaux moyens de développer les compétences à lire et à écrire d'un individu, lesquelles sont particulièrement sollicitées par certaines TIC comme l'ordinateur et Internet, et relativement moins pour l'usage du cellulaire, qui repose en grande partie sur l'oral et la numératie dans son utilisation de base. Par ailleurs, sans pouvoir les transposer de facto au cas du Mexique et du Guatémala, certaines études auprès de populations sud-américaines (par exemple Amiel, 2006, pour le cas du Brésil et Benitez, 2006, pour le cas des migrants salvadoriens de Washington D.C.) indiquent que l'école peut également représenter un lieu d'accès (parfois le seul) aux TIC. D'une manière générale, l'école développe aussi chez les individus des compétences cognitives leur permettant de traiter et, à ce titre, de tirer profit des informations accessibles par les TIC (George, 2004). Encore faut-il

${ }^{1}$ http://fr.wikipedia.org/wiki/Liste_des_pays_par_IDH\#IDH_2012 


\section{Simon COLLIN, Sirléia ROSA, Hamid SAFFARI et Gisèle CHARPENTIER}

reconnaitre que l'effet " éducation » n'est pas semblable chez tous ceux qui y ont accès, même au sein d'un système éducatif unique et à niveau éducatif égal (Hargittai, 2010).

Il est intéressant de noter que l'âge ne ressort pas comme une variable significative au sein de notre échantillon, ce qui va à l'encontre des résultats de certaines études, telles que celles de Codganone et Kluzer (2011) et Benítez (2006). En revanche, plusieurs autres études ont déjà mis de l'avant que l'âge ne saurait être la seule variable explicative du rapport aux TIC et que d'autres, notamment celles trouvées ici, expliquent autant, si ce n'est plus, les usages des TIC par les individus (voir par exemple Hargittai, 2010; Helsper et Eynon, 2010; Livingstone et Helsper, 2007).

En résumé, les usages des TIC par les travailleurs migrants temporaires semblent davantage s'expliquer par une tendance à la reproduction des usages préexistants dans la société d'origine. Dans cette logique, les travailleurs migrants temporaires semblent rester en contact avec leurs proches en utilisant la ou les TIC qu'ils utilisaient déjà en phase de non-migration, en premier lieu le téléphone cellulaire. Rester en contact avec ses proches ne semble donc pas être une motivation à utiliser davantage de TIC, dès lors que le minimum technologique nécessaire est déjà disponible. Ce résultat semble rejoindre l'étude ethnographique de Komito et Bates (2011). Ces auteurs montrent qu'Internet est moins important que le réseau social comme source d'information sur le pays d'accueil, en phase prémigratoire, et qu'il ne gagne pas en importance en phase post-migratoire.

Globalement, les usages des TIC par les travailleurs migrants temporaires de cette étude sont limités par rapport à ceux de la population québécoise, si l'on en croit quelques rapports récents tels que celui du CEFRIO (2011), qui dresse un portrait des usages des TIC de cinq générations de la population du Québec. Dans la mesure où l'hétérogénéité des usages entre les participants s'explique possiblement par des variables liées à la nationalité, que nous lions au développement infrastructurel de la société d'origine, et au niveau d'éducation, les usages des TIC par les travailleurs migrants temporaires nous semble indissociables de la problématique des inégalités socionumériques (Hargittai et Hsieh, 2013). À l'inverse, suivant la thèse de l'empowerment, il aurait été attendu qu'ils mettent davantage à profit les diverses opportunités relationnelles permises par les TIC pour rester en contact avec leurs proches. 
Les travailleurs migrants temporaires au Québec : des

migrants connectés?

Les résultats présentés ne peuvent cependant en aucun être généralisés à l'ensemble de la population des travailleurs migrants temporaires. En effet, une limite évidente a trait à la taille de l'échantillon et à ses implications sur les tests statistiques effectués. Par ailleurs, la ferme dans laquelle s'est déroulée cette recherche est vraisemblablement soucieuse des conditions de vie de ses travailleurs migrants temporaires, comme le montrent plusieurs projets effectués récemment ou projetés à court terme (citons, à titre d'exemples, l'aménagement d'une cafétéria, la rénovation des logements, la mise à disposition d'une boîte aux lettres anonymes pour que les travailleurs puissent s'exprimer plus librement auprès de la direction, etc.). II est difficile de savoir dans quelle mesure ces initiatives louables sont représentatives de l'ensemble des employeurs des travailleurs migrants temporaires au Canada.

En termes de pistes de recherches futures, il semblerait intéressant de continuer à étudier les usages des TIC par les travailleurs migrants temporaires sur des échantillons plus larges. Par ailleurs, comme la variable migratoire semble avoir relativement peu d'influence sur les usages des TIC par les participants à l'étude, il serait bon de préciser son rôle en relation aux autres variables socioculturelles. II serait également intéressant, dans une visée comparative, d'étudier plus en détail les divergences et les convergences des usages des TIC par cette population au regard des autres groupes migrants et ethniques. D'une manière plus globale, le rapport et les liens entre les différentes dimensions explorées ici méritent davantage d'attention de la part des chercheurs de manière à mieux comprendre comment se développe et évolue le rapport des individus aux TIC.

\section{Références bibliographiques}

AMAR M., G. ROBERGE, A. LARUE, L. GÉLINEAU et Y. LEANZA, 2009, Rapport de recherche-évaluation: les travailleurs agricoles migrants mexicains et guatémaltèques de l'île d'Orléans, Centre de santé et des services sociaux de la Veille-Capitale.

AMIEL T., 2006, « Mistaking computer for technology: Technology literacy and digital divide », AACE Journal, vol.14, pp.235-256.

BASOK T., 2000, «Migration of Mexican Seasonal Farm Workers to Canada and Development: Obstacles to Productive 
Simon COLLIN, Sirléia ROSA, Hamid SAFFARI et Gisèle CHARPENTIER

Investment », International Migration Review, vol.34, n91, pp.7997.

BAYON S., 2009, " Les TIC dans les collectifs diasporiques: étude des Bretons à New York », tic\&société, vol.3, n91, pp.75197.

BELLEROSE J. et C. YORN, 2013, Les travailleurs migrants sur les fermes du Québec: rapport de recherche, Centre d'innovation sociale en agriculture.

BENITEZ J. L., 2006, « Transnational dimensions of the diaital divide amona Salvadoran immigrants in the Washinaton DC metropolitan area », Global Networks, vol.2, n6, pp.181-199.

BINFORD L., 2006, « Campos agricolas, campos de poder: el estado mexicano, los granjeros canadienses y los trabajadores temporales mexicanos ", Migraciones Internacionales, vol.3, n33, pp.54-80.

BORKERT M., P. CINGOLANI et V. PREMAZZI, 2009, The State of the Art of Research in the EU on the Take up and Use of ICT by Immigrants and Ethnic Minorities, Luxembourg, Office for Official Publications of the European Communities.

CAIDI N. et D. ALLARD, 2005, "Social inclusion of newcomers to Canada: An information problem? », Library \& Information Science Research, vol.27, n33, pp.302-324.

CEFRIO, 2011, Cinq générations d'internautes: profil d'utilisation des TIC en 2011, vol.2, $\mathrm{n} \%$, <http://www.cefrio.qc.ca/media/uploader/2_generations.pdf>, dernière consultation le 27 juin 2014.

CHINN M.D. et R.W. FAIRLIE, 2007, « The determinants of the global digital divide: a cross-country analysis of computer and internet penetration », Oxford Economic Papers, vol.59, no1, pp.16-44.

CODAGNONE C. et S. KLUZER, 2011, ICT for the Social and Economic Integration of Migrants into Europe, Luxembourg, Office for Official Publications of the European Communities.

COLLIN S., 2012, "ICTs and Migration: The Mapping of an Emerging Area of Research ». The International Journal of Technology, Knowledge and Society, vol.8, n2, pp.65-78.

DIMAGGIO P., E. HARGITTAI, C. CELESTE et S. SHAFER, 2004, «Digital Inequality: From Unequal Access to 
Les travailleurs migrants temporaires au Québec : des

$$
\text { migrants connectés? }
$$

Differenciated Use ", dans K. M. Neckerman (Dir.), Social Inequality, New York, Russel Sage Fundation, pp. 355-400.

DIMINESCU D., 2002, «Les migrations à l'âge des nouvelles technologies ", Revue Hommes et Migrations, vol.1240, n2, pp.6-9.

DIMINESCU D., 2005, " Le migrant connecté : pour un manifeste épistémologique ", Migrations Société, vol.17, n`102, pp. 275-292.

FIBBI, R et G. D'AMATO, 2008, "Transnationalisme des migrants en Europe : une preuve par les fait ", Revue Européenne des Migrations Internationales, vol.24, n2, pp.722.

FOND MONÉTAIRE INTERNATIONAL, 2013, World Economic outlook : Hopes, Realities, Risks, <http://www.imf.org/external/pubs/ft/weo/2013/01/pdf/text.pdf>, dernière consultation le 27 juin 2014.

GARDUÑO, E., 2003, "Antropologia de la frontera, la migraciôn y los procesos transnacionales ", Frontera Norte, vol.15, n30, pp.65-89.

George, É., 2004, «L'expression de la fracture numérique en question ", Dans É. Guichard (Dir.), Mesures de l'Internet, Paris, France, Éditions des Canadiens en Europe.

HANAFIZADEH M. R., A. SAGHAEI et P. HANAFIZADEH, 2009, " An index for cross-country analysis of ICT infrastructure and access ", Telecommunications Policy, vol.33, n7, pp.385405.

HANNAFORD D., 2014, "Technologies of the spouse: intimate surveillance in Senegalese transnational marriages ", Global Networks, doi: 10.1111/glob.12045

HARGITTAI E., 2010, « Digital Na(t)ives? Variation in Internet skills and uses among members ofthe "Net Generation" ", Sociological Inquiry, vol.80, $n$ ㅇ, pp.92-113.

HARGITTAI E. et Y. HSIEH, 2013, « Digital inequality », dans W. Dutton, The Oxford Handbook of Internet Studies, Oxford, Oxford University Press, pp.129-150.

HELSPER E.J. et R. EYNON, 2010, " Digital natives:Where is the evidence? ", British Educational Research Journal, vol.36, nऊ, pp.503-520. 


\section{Simon COLLIN, Sirléia ROSA, Hamid SAFFARI et Gisèle}

CHARPENTIER

HILLER H.H. et T.M. FRANZ, 2004, « New ties, old ties and lost ties: the use of the internet in diaspora », New Media \& Society, vol.6, n6, pp.731-752.

INTERNATIONAL TELECOMMUNICATION UNION (ITU), 2013, The World in 2013: ICT, Facts and Figures, < http://www.itu.int/en/ITUD/Statistics/Documents/facts/ICTFactsF igures2013.pdf>, dernière consultation le 01 juin 2014.

KLUZER S., A. HACHE et C. CODAGNONE, 2008, Overview of Digital Support Initiatives for/by Immigrants and Ethnic Minorities in the EU27, Luxembourg, Office for Official Publications of the European Communities.

KOMITO L., et J. BATES, 2011, «Migrants' information practices and use of social media in Ireland: networks and community », iConference 2011, Seattle, USA, pp. 289-295.

LIVINGSTONE S., et E. HELSPER, 2007, «Gradations in digital inclusion: children, young people and the digital divide », New Media \& Society, vol.9, n4, pp.671-696.

MATTELART T., 2009, «Les diasporas à l'heure des technologies de l'information et de la communication: petit état des savoirs », tic\&société, vol.3, n91-2, pp.11-56.

MRAD F., 2006, «Transfert technologique et capacité d'absorption: cas d'un pays en développement », dans C. Ammi, Innovations technologiques: aspects culturels et mondialisation, Paris, Lavoisier, pp. 59-72.

NEDELCU M. F., 2002, « L'utilisation d' un espace virtuel par une communauté de professionnels immigrés : vers une nouvelle forme d' organisation diasporique? ", Revue de sciences sociales au Sud, vol.22, pp.147-165.

NEDELCU M. F., 2009, Nouveaux moyens de communication, nouveaux espaces sociaux, Le transnationalisme à l'ère du migrant online, Université de Neuchâtel Unine, Editions L'Harmattan,

<www.ekm.admin.ch/de/aktuell/.../ref_nedelcu.pdf>, dernière consultation le 27 juin 2014.

OOSTERBAAN M., 2010, «Virtual Migration. Brazilian Diasporic Media and the Reconfigurations of Place and Space», Revue européenne des migrations internationales, vol.26, $\mathrm{n}$ ㅇ, pp.81-102. 
Les travailleurs migrants temporaires au Québec : des

migrants connectés?

PORTES A, W. HALLER et L. GUARNIZO, 2002, «Transnational Entrepreneurs: The Emergence and Determinants of an Alternative Form of Immigrant Economic Adaptation », American Sociological Review, vol. 67, n, pp. 278-298.

PREIBISCH K. L., 2007, «Local Produce, Foreign Labor: Labor Mobility Programs and Global Trade Competitiveness in Canada », Rural Sociology, vol.72, n3, pp.418-449.

RESSOURCES HUMAINES ET DEVELOPPEMENT DES COMPETENCES CANADA, 2009, Programme des travailleurs agricoles saisonniers. Ottawa: Gouvernement du Canada, $<$ http://www.rhdcc.gc.ca/fra/emplois/travailleurs_etrangers/inde x.shtml>, dernière consultation le 27 juin 2014.

REYES TRIGOS C., 2006, «La dinámica familiar y el Programa de Trabajadores Agrícolas Temporales MéxicoCanadá », UAM Iztapalapa, vol.60, pp.107-124.

RIGONI I., 2010, «Les médias des minorités ethniques. Réprésenter l'identité collective sur la scène publique ", Revue européenne des migrations internationales, vol.26, pp.6-16.

ROS A., 2010, « Interconnected Immigrants in the Information Society », dans A. Alonso et P. Oiarzabal (dir.), Diasporas in the New Media Age: Identity, Politics, and Community, Reno, University of Nevada Press, pp. 19-39.

SCOPSI C., 2004, « Représentation des TIC et multiterritorialité : le cas des télé et cyberboutiques de Château rouge, à Paris", dans A. CHENEAU- LOQUAY (dir), Mondialisation et technologies de la communication en Afrique, Paris-Pessac, Karthala-MSHA.

SCOPSI C., 2009, « Les sites Web diasporiques: un nouveau genre médiatique? », tic\&société, vol.3, n-1-2, pp.81-100.

STATISTIC Canada, 2011, Canadian Internet Use Survey, 2010, Questionnaire. Ottawa: Statistics Canada. <http://www23.statcan.gc.ca/imdbbmdi/instrument/4432_Q1_V9 -eng.htm>, dernière consultation le 27 juin 2014.

VICENTE M R et A. LOPEZ, 2006, «Patterns of ICT diffusion across the European Union », Economics Letters, vol.93, n91, pp.45-51.

WEI L., 2012, « Number Matters: The Multimodality of Internet Use as an Indicator of the Digital Inequalities », Journal of Computer-Mediated Communication, vol. 7, n2, pp.303-318. 
Simon COLLIN, Sirléia ROSA, Hamid SAFFARI et Gisèle CHARPENTIER 